\title{
On the Operator $\circledast^{k}$ Related to Heat Equation
}

\author{
Wanchak Satsanit \\ Department of Mathematics, Regina Coeli College \\ 166 Charoenprathet Road Changkran \\ Chiangmai, 50100, Thailand \\ E-mail: aunphue@live.com \\ Amnuay Kananthai \\ Department of Mathematics, Chiangmai University \\ Chiang Mai 50200, Thailand
}

\section{Abstract}

In this paper, we study the equation

$$
\frac{\partial}{\partial t} u(x, t)+c^{2}(-\circledast)^{k} u(x, t)=0
$$

with the initial condition

$$
u(x, 0)=f(x)
$$

for $x \in \mathbb{R}^{n}$-the $n$-dimensional Euclidean space. The operator $(\circledast)^{k}$ is operator iterated $k$ times, defined by

$$
\circledast^{k}=\left(\left(\sum_{i=1}^{p} \frac{\partial^{2}}{\partial x_{i}^{2}}\right)^{3}+\left(\sum_{j=p+1}^{p+q} \frac{\partial^{2}}{\partial x_{j}^{2}}\right)^{3}\right)^{k}
$$

$p+q=n$ is the dimension of the Euclidean space $\mathbb{R}^{n}, u(x, t)$ is an unknown function for $(x, t)=\left(x_{1}, x_{2}, \ldots, x_{n}, t\right) \in$ $\mathbb{R}^{n} \times(0, \infty), f(x)$ is the given generalized function, $k$ is a positive integer and $c$ is a positive constant. Moreover, if we put $q=0$ and $k=1$ we obtain the solution of equation.

$$
\frac{\partial}{\partial t} u(x, t)-c^{2} \triangle^{3} u(x, t)=0
$$

Which is related to the triharmonic heat equation.

Keywords: Fourier transform, Temper distribution, Diamond operator

\section{Introduction}

It is well known that for the heat equation

$$
\frac{\partial}{\partial t} u(x, t)=c^{2} \Delta u(x, t)
$$

with the initial condition

$$
u(x, 0)=f(x)
$$

where $\Delta=\sum_{i=1}^{n} \frac{\partial^{2}}{\partial x_{i}^{2}}$ is the Laplace operator and $(x, t)=\left(x_{1}, x_{2}, \ldots, x_{n}, t\right) \in \mathbb{R}^{n} \times(0, \infty)$, we obtain

$$
u(x, t)=\frac{1}{\left(4 c^{2} \pi t\right)^{n / 2}} \int_{\mathbb{R}^{n}} \exp \left(-\frac{|x-y|^{2}}{4 c^{2} t}\right) f(y) d y
$$

as the solution of (1).

Now, (2) can be written $u(x, t)=E(x, t) * f(x)$ where

$$
E(x, t)=\frac{1}{\left(4 c^{2} \pi t\right)^{n / 2}} \exp \left(-\frac{|x|^{2}}{4 c^{2} t}\right) .
$$

$E(x, t)$ is called the heat kernel, where $|x|^{2}=x_{1}^{2}+x_{2}^{2}+\cdots+x_{n}^{2}$ and $t>0$, see (F. John, p208-209). 
In 1996, A. Kananthai (1997) has introduced the Diamond operator $\diamond$ defined by

$$
\diamond=\left(\sum_{i=1}^{p} \frac{\partial^{2}}{\partial x_{i}^{2}}\right)^{2}-\left(\sum_{j=p+1}^{p+q} \frac{\partial^{2}}{\partial x_{j}^{2}}\right)^{2}, p+q=n
$$

or $\diamond$ can be written as the product of the operators in the form $\diamond=\Delta \square=\square \Delta$ where $\Delta=\sum_{i=1}^{n} \frac{\partial^{2}}{\partial x_{i}^{2}}$ is the Laplacian and $\square=\sum_{i=1}^{p} \frac{\partial^{2}}{\partial x_{i}^{2}}-\sum_{j=p+1}^{p+q} \frac{\partial^{2}}{\partial x_{j}^{2}}$ is the ultra-hyperbolic. The Fourier transform of the Diamond operator also has been studied and the elementary solution of such operator, see (A. Kananthai, 1999).

Next, K. Nonlaopon and A. Kananthai (2003) study the equation

$$
\frac{\partial}{\partial t} u(x, t)=c^{2} \square u(x, t)
$$

We obtain the ultra-hyperbolic heat kernel

$$
E(x, t)=\frac{i^{q}}{\left(4 c^{2} \pi t\right)^{n / 2}} \exp \left(-\frac{\sum_{i=1}^{p} x_{i}^{2}-\sum_{j=p+1}^{p+q} x_{j}^{2}}{4 c^{2} t}\right) .
$$

Next, W. Satsanit has been first introduced the $\circledast^{k}$ operator where $\circledast^{k}$ defined by

$$
\begin{aligned}
\circledast^{k} & =\left(\left(\sum_{i=1}^{p} \frac{\partial^{2}}{\partial x_{i}^{2}}\right)^{3}+\left(\sum_{j=p+1}^{p+q} \frac{\partial^{2}}{\partial x_{j}^{2}}\right)^{3}\right)^{k} \\
& =\left(\sum_{i=1}^{p} \frac{\partial^{2}}{\partial x_{i}^{2}}+\sum_{j=p+1}^{p+q} \frac{\partial^{2}}{\partial x_{j}^{2}}\right)^{k}\left[\left(\sum_{i=1}^{p} \frac{\partial^{2}}{\partial x_{i}^{2}}\right)^{2}-\left(\sum_{i=1}^{p} \frac{\partial^{2}}{\partial x_{i}^{2}}\right)\left(\sum_{j=p+1}^{p+q} \frac{\partial^{2}}{\partial x_{j}^{2}}\right)+\left(\sum_{j=p+1}^{p+q} \frac{\partial^{2}}{\partial x_{j}^{2}}\right)^{2}\right]^{k} \\
& =(\Delta)^{k}\left(\Delta^{2}-\frac{3}{4}(\Delta+\square)(\Delta-\square)\right)^{k} \\
& =\left(\frac{3}{4} \diamond \square+\frac{1}{4} \Delta^{3}\right)^{k}
\end{aligned}
$$

where

$$
\begin{aligned}
\Delta & =\frac{\partial^{2}}{\partial x_{1}^{2}}+\frac{\partial^{2}}{\partial x_{2}^{2}}+\ldots+\frac{\partial^{2}}{\partial x_{n}^{2}} \\
\square & =\frac{\partial^{2}}{\partial x_{1}^{2}}+\frac{\partial^{2}}{\partial x_{2}^{2}}+\cdots+\frac{\partial^{2}}{\partial x_{p}^{2}}-\frac{\partial^{2}}{\partial x_{p+1}^{2}}-\frac{\partial^{2}}{\partial x_{p+2}^{2}}-\cdots-\frac{\partial^{2}}{\partial x_{p+q}^{2}} \\
\diamond & =\left(\frac{\partial^{2}}{\partial x_{1}^{2}}+\frac{\partial^{2}}{\partial x_{2}^{2}}+\cdots+\frac{\partial^{2}}{\partial x_{p}^{2}}\right)^{2}-\left(\frac{\partial^{2}}{\partial x_{p+1}^{2}}+\frac{\partial^{2}}{\partial x_{p+2}^{2}}+\cdots+\frac{\partial^{2}}{\partial x_{p+q}^{2}}\right)^{2}
\end{aligned}
$$

Now, the purpose of this work is to study the equation

with the initial condition

$$
\frac{\partial}{\partial t} u(x, t)+c^{2}(-\circledast)^{k} u(x, t)=0
$$

for $x \in \mathbb{R}^{n}$-the $n$-dimensional Euclidean space. The $\circledast^{k}$ operator is defined by

$$
\begin{aligned}
\circledast^{k} & =\left(\left(\sum_{i=1}^{p} \frac{\partial^{2}}{\partial x_{i}^{2}}\right)^{3}+\left(\sum_{j=p+1}^{p+q} \frac{\partial^{2}}{\partial x_{j}^{2}}\right)^{3}\right)^{k} \\
& =\left(\sum_{i=1}^{p} \frac{\partial^{2}}{\partial x_{i}^{2}}+\sum_{j=p+1}^{p+q} \frac{\partial^{2}}{\partial x_{j}^{2}}\right)^{k}\left[\left(\sum_{i=1}^{p} \frac{\partial^{2}}{\partial x_{i}^{2}}\right)^{2}-\left(\sum_{i=1}^{p} \frac{\partial^{2}}{\partial x_{i}^{2}}\right)\left(\sum_{j=p+1}^{p+q} \frac{\partial^{2}}{\partial x_{j}^{2}}\right)+\left(\sum_{j=p+1}^{p+q} \frac{\partial^{2}}{\partial x_{j}^{2}}\right)^{2}\right]^{k} \\
& =(\triangle)^{k}\left(\Delta^{2}-\frac{3}{4}(\Delta+\square)(\Delta-\square)\right)^{k} \\
& =\left(\frac{3}{4} \diamond \square+\frac{1}{4} \triangle^{3}\right)^{k}
\end{aligned}
$$


$p+q=n$ is the dimension of the Euclidean space $\mathbb{R}^{n}, u(x, t)$ is an unknown function for $(x, t)=\left(x_{1}, x_{2}, \ldots, x_{n}, t\right) \in$ $\mathbb{R}^{n} \times(0, \infty), f(x)$ is the given generalized function and $c$ is a positive constant. We obtain $u(x, t)=E(x, t) * f(x)$ as a solution of (5), where

$$
E(x, t)=\frac{1}{(2 \pi)^{n}} \int_{R^{n}} \exp \left[-c^{2}\left[\left(\sum_{i=1}^{p} \xi_{i}^{2}\right)^{3}+\left(\sum_{j=p+1}^{p+q} \xi_{j}^{2}\right)^{3}\right]^{k} t+i(\xi, x)\right] d \xi
$$

The function $E(x, t)$ is the elementary solution of (5).

All properties of $E(x, t)$ will be studied in details.

Now, if we put $q=0$ and $k=1$ in (5), then (5) reduces to the equation

$$
\frac{\partial}{\partial t} u(x, t)-c^{2} \Delta^{3} u(x, t)=0
$$

which is related to the triharmonic heat equation.

Before going that points, the following definitions and some concepts are needed.

\section{Preliminaries}

Definition 2.1 Let $f(x) \in \mathrm{L}_{1}\left(\mathbb{R}^{n}\right)$-the space of integrable function in $\mathbb{R}^{n}$. The Fourier transform of $f(x)$ is defined by

$$
\widehat{f}(\xi)=\frac{1}{(2 \pi)^{n / 2}} \int_{\mathbb{R}^{n}} e^{-i(\xi, x)} f(x) d x
$$

where $\xi=\left(\xi_{1}, \xi_{2}, \ldots, \xi_{n}\right)$ and $x=\left(x_{1}, x_{2}, \ldots, x_{n}\right) \in \mathbb{R}^{n},(\xi, x)=\xi_{1} x_{1}+\xi_{2} x_{2}+\cdots+\xi_{n} x_{n}$ and $d x=d x_{1} d x_{2} \ldots d x_{n}$.

Also, the inverse of Fourier transform is defined by

$$
f(x)=\frac{1}{(2 \pi)^{n / 2}} \int_{\mathbb{R}^{n}} e^{i(\xi, x)} \widehat{f}(\xi) d \xi .
$$

If $f$ is a distribution with compact supports $f$ can be written as (See A. H. Zemanian, 1965, Theorem 7.4-3, p.187 Eq.(2.1))

$$
\widehat{f}(\xi)=\frac{1}{(2 \pi)^{n / 2}}\left\langle f(x), e^{-i(\xi, x)}\right\rangle .
$$

Lemma 1 Given the function

$$
f(x)=\exp \left(-\left(\left(\sum_{i=1}^{p} x_{i}^{2}\right)^{3}+\left(\sum_{j=p+1}^{p+q} x_{j}^{2}\right)^{3}\right)^{k}\right)
$$

where

$$
\left(x_{1}, x_{2}, \ldots, x_{n}\right) \in \mathbb{R}^{n}, \quad p+q=n,
$$

and $k$ is the positive number. Then

$$
\left|\int_{\mathbb{R}^{n}} f(x) d x\right| \leq \frac{\pi^{n / 2}}{9 k^{2}} \cdot \frac{\Gamma\left(\frac{p}{6 k}\right) \Gamma\left(\frac{q}{6 k}\right)}{\Gamma\left(\frac{p}{2}\right) \Gamma\left(\frac{q}{2}\right)}
$$

where $\frac{p+q}{2}=\frac{n}{2}$ and $\Gamma$ denoted the gamma function. That is $\int_{\mathbb{R}^{n}} f(x) d x$ is bounded.

Proof.

$$
\int_{\mathbb{R}^{n}} f(x) d x=\int_{\mathbb{R}^{n}} \exp \left(-\left(\left(\sum_{i=1}^{p} x_{i}^{2}\right)^{3}+\left(\sum_{j=p+1}^{p+q} x_{j}^{2}\right)^{3}\right)^{k}\right) d x
$$

for $k$ is a positive integer. By changing the coordinate.Now , put

$$
\begin{gathered}
x_{1}=y_{1}, \quad x_{2}=y_{2}, \ldots, \quad x_{p}=y_{p} \\
d x_{1}=d y_{1}, \quad d x_{2}=d y_{2}, \ldots, \quad d x_{p}=d y_{p}
\end{gathered}
$$

and

$$
x_{p+1}=y_{p+1}, \quad x_{p+2}=y_{p+2}, \ldots, \quad x_{p+q}=y_{p+q}
$$


Then we obtain

$$
d x_{p+1}=d y_{p+1}, d x_{p+2}=d y_{p+2}, \ldots, d x_{p+q}=d y_{p+q}
$$

$$
\int_{\mathbb{R}^{n}} f(x) d x=\int_{\mathbb{R}^{n}} \exp \left(-\left(\left(\sum_{j=p+1}^{p+q} y_{j}^{2}\right)^{3}+\left(\sum_{i=1}^{p} y_{i}^{2}\right)^{3}\right)^{k}\right) d y
$$

Let us transform to bipolar coordinates defined by

$$
y_{1}=r w_{1}, y_{2}=r w_{2}, \ldots, y_{p}=r w_{p}
$$

and

$$
y_{p+1}=s w_{p+1}, y_{p+2}=s w_{p+2}, \ldots, y_{p+q}=s w_{p+q}, \quad p+q=n
$$

where $w_{1}^{2}+w_{2}^{2}+\cdots+w_{p}^{2}=1$ and $w_{p+1}^{2}+w_{p+2}^{2}+\cdots+w_{p+q}^{2}=1$, Thus

$$
\int_{\mathbb{R}^{n}} f(x) d x=\int_{\mathbb{R}^{n}} \exp \left(-\left(r^{6}+s^{6}\right)^{k}\right) r^{p-1} s^{q-1} d r d s d \Omega_{p} d \Omega_{q}
$$

where

$$
d y=r^{p-1} s^{q-1} d r d s d \Omega_{p} d \Omega_{q}
$$

$d \Omega_{p}$ and $d \Omega_{q}$ are the elements of surface area on the unit sphere in $R^{p}$ and $R^{q}$ respectively.By computing directly , we obtain

$$
\int_{\mathbb{R}^{n}} f(x) d x=\Omega_{p} \Omega_{q} \int_{0}^{\infty} \int_{0}^{\infty} \exp \left(-\left(r^{6}+s^{6}\right)^{k}\right) r^{p-1} s^{q-1} d r d s
$$

where $\Omega_{p}=\frac{2 \pi^{p / 2}}{\Gamma(p / 2)}$ and $\Omega_{q}=\frac{2 \pi^{q / 2}}{\Gamma(q / 2)}$.

Since $\left(r^{6}+s^{6}\right)^{k} \geq r^{6 k}+s^{6 k}$, then

$$
\exp \left(-\left(r^{6}+s^{6}\right)^{k}\right) \leq \exp \left(-\left(r^{6 k}+s^{6 k}\right)\right.
$$

Thus

$$
\begin{aligned}
\left|\int_{\mathbb{R}^{n}} f(x) d x\right| & \leq \Omega_{p} \Omega_{q} \int_{0}^{\infty} \int_{0}^{\infty} \exp \left(-r^{6 k}-s^{6 k}\right) r^{p-1} s^{q-1} d r d s \\
& =\Omega_{p} \Omega_{q} \int_{0}^{\infty} \exp \left(-r^{6 k}\right) r^{p-1} d r \int_{0}^{\infty} \exp \left(-s^{6 k}\right) s^{q-1} d s
\end{aligned}
$$

Put $u=r^{6 k}, d r=\frac{1}{6 k} u^{\frac{1}{6 k}-1} d u$ and $v=s^{6 k}, d s=\frac{1}{6 k} v^{\frac{1}{6 k}-1} d v$

Then we obtain

$$
\begin{aligned}
\left|\int_{\mathbb{R}^{n}} f(x) d x\right| & \leq \frac{\Omega_{p} \Omega_{q}}{(6 k)^{2}} \int_{0}^{\infty} e^{-u} u^{\frac{p}{6 k}-1} d u \int_{0}^{\infty} e^{-v} v^{\frac{q}{6 k}-1} d v \\
& =\frac{\Omega_{p} \Omega_{q}}{(6 k)^{2}} \Gamma\left(\frac{p}{6 k}\right) \Gamma\left(\frac{q}{6 k}\right) \\
& =\frac{2 \pi^{p / 2} 2 \pi^{q / 2}}{(6 k)^{2}} \frac{\Gamma\left(\frac{p}{6 k}\right) \Gamma\left(\frac{q}{6 k}\right)}{\Gamma\left(\frac{p}{2}\right) \Gamma\left(\frac{q}{2}\right)} \\
& =\frac{\pi^{n / 2} \Gamma\left(\frac{p}{6 k}\right) \Gamma\left(\frac{q}{6 k}\right)}{9 k^{2}} \frac{p}{\Gamma\left(\frac{p}{2}\right) \Gamma\left(\frac{q}{2}\right)}
\end{aligned}
$$

Where $\frac{p+q}{2}=\frac{n}{2}$. That is $\int_{\mathbb{R}^{n}} f(x) d x$ is bounded.

Lemma 2 (The Fourier transform of $(-\circledast)^{k} \delta$ )

$$
\mathcal{F}(-\circledast)^{k} \delta=\frac{(-1)^{4 k}}{(2 \pi)^{n / 2}}\left[\left(\xi_{1}^{2}+\xi_{2}^{2}+\ldots+\xi_{p}^{2}\right)^{3}+\left(\xi_{p+1}^{2}+\xi_{p+2}^{2}+\ldots+\xi_{p+q}^{2}\right)^{3}\right]^{k}
$$

where $\mathcal{F}$ is the Fourier transform defined by Eq.(7) and if the norm of $\xi$ is given by $\|\xi\|=\left(\xi_{1}^{2}+\xi_{2}^{2}+\ldots+\xi_{n}^{2}\right)^{1 / 2}$ then

$$
\mathcal{F}(-\circledast)^{k} \delta \leq \frac{3^{k}}{(2 \pi)^{n / 2}}\|\xi\|^{6 k}
$$

that is $\mathcal{F}(-\circledast)^{k}$ is bounded and continuous on the space $\mathcal{S}^{\prime}$ of the tempered distribution. Moreover, by Eq.(8)

$$
(-\circledast)^{k} \delta=\mathcal{F}^{-1} \frac{1}{(2 \pi)^{n / 2}}\left[\left(\xi_{1}^{2}+\xi_{2}^{2}+\ldots+\xi_{p}^{2}\right)^{3}+\left(\xi_{p+1}^{2}+\xi_{p+2}^{2}+\ldots+\xi_{p+q}^{2}\right)^{3}\right]^{k}
$$


Proof. By Eq. (9)

$$
\begin{aligned}
\mathcal{F}(-\circledast)^{k} \delta= & \frac{1}{(2 \pi)^{n / 2}}\left\langle(-\circledast)^{k} \delta, e^{-i(\xi \cdot x)}\right\rangle \\
= & \frac{1}{(2 \pi)^{n / 2}}\left\langle\delta,(-\circledast)^{k} e^{-i(\xi \cdot x)}\right\rangle \\
= & \frac{1}{(2 \pi)^{n / 2}}\left\langle\delta,(-\circledast)^{k-1}(-\circledast) e^{-i(\xi \cdot x)}\right\rangle \\
= & \frac{1}{(2 \pi)^{n / 2}}\left\langle\delta,(-\circledast)^{k-1}\left(-\frac{3}{4} \diamond \square-\frac{1}{4} \Delta^{3}\right) e^{-i(\xi \cdot x)}\right\rangle \\
= & \frac{1}{(2 \pi)^{n / 2}}\left\langle\delta,(-\circledast)^{k-1}\left(-\frac{3}{4} \diamond \square\right) e^{-i(\xi \cdot x)}\right\rangle+\frac{1}{(2 \pi)^{n / 2}}\left\langle\delta,(-\circledast)^{k-1}\left(-\frac{1}{4} \square^{3}\right) e^{-i(\xi \cdot x)}\right\rangle \\
= & \frac{1}{(2 \pi)^{n / 2}}\left\langle\delta,(-\circledast)^{k-1} \frac{3}{4}(-1)^{4}\left[\left(\sum_{i=1}^{p} \xi_{i}^{2}\right)^{2}-\left(\sum_{j=p+1}^{p+q} \xi_{j}^{2}\right)^{2}\right] .\right. \\
& \left.\left(\sum_{i=1}^{p} \xi_{i}^{2}-\sum_{j=p+1}^{p+q} \xi_{j}^{2}\right) e^{-i(\xi \cdot x)}\right\rangle+\frac{1}{(2 \pi)^{n / 2}}\left\langle\delta,(-\circledast)^{k-1} \frac{1}{4}(-1)^{4}\left(\sum_{i=1}^{n} \xi_{i}^{2}\right)^{3} e^{-i(\xi \cdot x)}\right\rangle \\
= & \frac{1}{(2 \pi)^{n / 2}}\left\langle\delta,(-\circledast)^{k-1} \frac{3}{4}(-1)^{4}\left[\left(\sum_{i=1}^{p} \xi_{i}^{2}\right)^{2}-\left(\sum_{j=p+1}^{p+q} \xi_{j}^{2}\right)^{2}\right]\left(\sum_{i=1}^{p} \xi_{i}^{2}-\sum_{j=p+1}^{p+q} \xi_{j}^{2}\right)\right. \\
= & \frac{(-1)^{4}}{(2 \pi)^{n / 2}}\left\langle\delta,(-1)^{4}\left(\sum_{i=1}^{n} \xi_{i}^{2}\right)^{3} e^{-i(\xi \cdot x)}\right\rangle
\end{aligned}
$$

By keeping on operator $(-\circledast)$ with $k-1$ times , we obtain

$$
\mathcal{F}(-\circledast)^{k} \delta=\frac{(-1)^{4 k}}{(2 \pi)^{n / 2}}\left[\left(\xi_{1}^{2}+\xi_{2}^{2}+\ldots+\xi_{p}^{2}\right)^{3}+\left(\xi_{p+1}^{2}+\xi_{p+2}^{2}+\ldots+\xi_{p+q}^{2}\right)^{3}\right]^{k}
$$

Now,

$$
\begin{aligned}
\left|\mathcal{F}(-\circledast)^{k} \delta\right| & =\frac{1}{(2 \pi)^{n / 2}}\left|\left(\xi_{1}^{2}+\xi_{2}^{2}+\ldots+\xi_{p}^{2}\right)^{3}+\left(\xi_{p+1}^{2}+\xi_{p+2}^{2}+\ldots+\xi_{p+q}^{2}\right)^{3}\right|^{k} \\
& \leq \frac{1}{(2 \pi)^{n / 2}}\left|\xi_{1}^{2}+\ldots+\xi_{n}^{2}\right|^{k}\left|\left(\xi_{1}^{2}+\ldots+\xi_{n}^{2}\right)^{2}+\left(\xi_{1}^{2}+\ldots+\xi_{n}^{2}\right)^{2}+\left(\xi_{1}^{2}+\ldots+\xi_{n}^{2}\right)^{2}\right|^{k} \\
& \leq \frac{3^{k}}{(2 \pi)^{n / 2}}\|\xi\|^{6 k}
\end{aligned}
$$

where $\|\xi\|=\left(\xi_{1}^{2}+\xi_{2}^{2}+\ldots+\xi_{n}^{2}\right)^{1 / 2}, \xi_{i}(i=1,2, \ldots, n) \in \mathbb{R}$. Hence we obtain $\mathcal{F}(-\circledast)^{k} \delta$ is bounded and continuous on the space $\mathcal{S}^{\prime}$ of the tempered distribution.

Since $\mathcal{F}$ is $1-1$ transformation from the space $\mathcal{S}^{\prime}$ of the tempered distribution to the real space $\mathbb{R}$, then by (8)

$$
\circledast \delta=\mathcal{F}^{-1} \frac{1}{(2 \pi)^{n / 2}}\left[\left(\xi_{1}^{2}+\xi_{2}^{2}+\ldots+\xi_{p}^{2}\right)^{3}+\left(\xi_{p+1}^{2}+\xi_{p+2}^{2}+\ldots+\xi_{p+q}^{2}\right)^{3}\right] .
$$

That completes the proof.

\section{Main Results}

Theorem 1 Given the equation

$$
\frac{\partial}{\partial t} u(x, t)+c^{2}(-\circledast)^{k} u(x, t)=0
$$

with the initial condition

$$
u(x, 0)=f(x)
$$


The operator

$$
\begin{aligned}
(\circledast)^{k} & =\left(\left(\sum_{i=1}^{p} \frac{\partial^{2}}{\partial x_{i}^{2}}\right)^{3}+\left(\sum_{j=p+1}^{p+q} \frac{\partial^{2}}{\partial x_{j}^{2}}\right)^{3}\right)^{k} \\
& =\left(\sum_{i=1}^{p} \frac{\partial^{2}}{\partial x_{i}^{2}}+\sum_{j=p+1}^{p+q} \frac{\partial^{2}}{\partial x_{j}^{2}}\right)^{k}\left[\left(\sum_{i=1}^{p} \frac{\partial^{2}}{\partial x_{i}^{2}}\right)^{2}-\left(\sum_{i=1}^{p} \frac{\partial^{2}}{\partial x_{i}^{2}}\right)\left(\sum_{j=p+1}^{p+q} \frac{\partial^{2}}{\partial x_{j}^{2}}\right)+\left(\sum_{j=p+1}^{p+q} \frac{\partial^{2}}{\partial x_{j}^{2}}\right)^{2}\right]^{k} \\
& =\left(\frac{3}{4} \diamond \square+\frac{1}{4} \Delta^{3}\right)^{k}
\end{aligned}
$$

$p+q=n$ is the dimension of Euclidean space $\mathbb{R}^{n}, k$ is a positive integer, $u(x, t)$ is an unknown function for $(x, t)=$ $\left(x_{1}, x_{2}, \ldots, x_{n}, t\right) \in \mathbb{R}^{n} \times(0, \infty), f(x)$ is the given generalized function, and $c$ is a positive constant. Then we obtain

$$
E(x, t)=\frac{1}{(2 \pi)^{n}} \int_{R^{n}} \exp \left[-c^{2}\left[\left(\sum_{i=1}^{p} \xi_{i}^{2}\right)^{3}+\left(\sum_{j=1}^{p+q} \xi_{j}^{2}\right)^{3}\right]^{k} t+i(\xi, x)\right] d \xi
$$

as a elementary solution of (13).

Proof. Taking the Fourier transform defined by (7) to both sides of (13), we obtain

$$
\frac{\partial}{\partial t} \widehat{u}(\xi, t)+c^{2}\left[\left(\sum_{i=1}^{p} \xi_{i}^{2}\right)^{3}+\left(\sum_{j=p+1}^{p+q} \xi_{j}^{2}\right)^{3}\right]^{k} \widehat{u}(\xi, t)=0
$$

(see Lemma 8). Thus

$$
\widehat{u}(\xi, t)=K(\xi) \exp \left[-c^{2} t\left(\left(\sum_{i=1}^{p} \xi_{i}^{2}\right)^{3}+\left(\sum_{j=p+1}^{p+q} \xi_{j}^{2}\right)^{3}\right)^{k}\right]
$$

where $K(\xi)$ is constant and $\widehat{u}(\xi, 0)=K(\xi)$.

Now, for $k$ is positive number.Thus $\widehat{u}(\xi, t)$ in (16) is bound and can be written by

$$
\widehat{u}(\xi, t)=K(\xi) \exp \left[-c^{2} t\left(\left(\sum_{i=1}^{p} \xi_{i}^{2}\right)^{3}+\left(\sum_{j=p+1}^{p+q} \xi_{j}^{2}\right)^{3}\right)^{k}\right]
$$

Now, by (14) we have

$$
K(\xi)=\widehat{u}(\xi, 0)=\widehat{f}(\xi)=\frac{1}{(2 \pi)^{n / 2}} \int_{\mathbb{R}^{n}} e^{-i(\xi, x)} f(x) d x
$$

and by the inversion (8)in (17) and (18) we obtain

$$
\begin{aligned}
u(x, t) & =\frac{1}{(2 \pi)^{n / 2}} \int_{\mathbb{R}^{n}} e^{i(\xi, x)} \widehat{u}(\xi, t) d \xi \\
& =\frac{1}{(2 \pi)^{n}} \int_{\mathbb{R}^{n}} \int_{\mathbb{R}^{n}} e^{i(\xi, x)} e^{-i(\xi, y)} f(y) \exp \left[-c^{2} t\left(\left(\sum_{i=1}^{p} \xi_{i}^{2}\right)^{3}+\left(\sum_{j=p+1}^{p+q} \xi_{j}^{2}\right)^{3}\right)^{k}\right] d y d \xi
\end{aligned}
$$

Thus

$$
u(x, t)=\frac{1}{(2 \pi)^{n}} \int_{\mathbb{R}^{n}} \int_{\mathbb{R}^{n}} e^{i(\xi, x-y)} \exp \left[-c^{2} t\left(\left(\sum_{i=1}^{p} \xi_{i}^{2}\right)^{3}+\left(\sum_{j=p+1}^{p+q} \xi_{j}^{2}\right)^{3}\right)^{k}\right] f(y) d y d \xi
$$

or

$$
u(x, t)=\frac{1}{(2 \pi)^{n}} \int_{\mathbb{R}^{n}} \int_{\mathbb{R}^{n}} \exp \left[-c^{2} t\left[\left(\sum_{i=1}^{p} \xi_{i}^{2}\right)^{3}+\left(\sum_{j=p+1}^{p+q} \xi_{j}^{2}\right)^{3}\right]^{k}+i(\xi, x-y)\right] f(y) d y d \xi
$$

Set

$$
E(x, t)=\frac{1}{(2 \pi)^{n}} \int_{\mathbb{R}^{n}} \exp \left[-c^{2} t\left[\left(\sum_{i=1}^{p} \xi_{i}^{2}\right)^{3}+\left(\sum_{j=p+1}^{p+q} \xi_{i}^{2}\right)^{3}\right]^{k}+i(\xi, x)\right] d \xi
$$


Thus (19) can be written in the convolution form

$$
u(x, t)=E(x, t) * f(x) .
$$

Now, the E(x,t) given by (15) exists.

We obtain $u(x, t)=E(x, t) * f(x)$ is a solution of $(13)$, where $E(x, t)$ is defined by $(15)$. and $k$ is positive integer.

Theorem 2 The kernel E(x,t) defined by (15) has the following properties :

(1) $E(x, t) \in C^{\infty}$-the space of continuous function for $x \in \mathbb{R}^{n}, t>0$ with infinitely differentiable.

(2) $\left(\frac{\partial}{\partial t}+c^{2}(-\circledast)^{k}\right) E(x, t)=0$ for $t>0$.and $k$ is positive integer.

(3) $E(x, t)>0$ for $t>0$.

(4) $|E(x, t)| \leq \frac{1}{9.2^{n} \pi^{n / 2} k^{2}\left(c^{2} t\right)^{\frac{n}{6 k}}} \frac{\Gamma\left(\frac{p}{6 k}\right) \Gamma\left(\frac{q}{6 k}\right)}{\Gamma\left(\frac{p}{2}\right) \Gamma\left(\frac{q}{2}\right)}$, for $t>0$,

Thus $E(x, t)$ is bounded for any fixed $t>0$.

(5) $\lim _{t \rightarrow 0} E(x, t)=\delta$.

\section{Proof.}

(1) From (15), since

$$
\frac{\partial^{n}}{\partial x^{n}} E(x, t)=\frac{1}{(2 \pi)^{n}} \int_{R^{n}} \frac{\partial^{n}}{\partial x^{n}} \exp \left[-c^{2}\left[\left(\sum_{i=1}^{p} \xi_{i}^{2}\right)^{3}+\left(\sum_{j=1}^{p+q} \xi_{j}^{2}\right)^{3}\right]^{k} t+i(\xi, x)\right] d \xi
$$

Thus $E(x, t) \in C^{\infty}$ for $x \in \mathbb{R}^{n}, t>0$.

(2) By computing directly, we obtain

$$
\left(\frac{\partial}{\partial t}+c^{2}(-\circledast)^{k}\right) E(x, t)=0
$$

for $t>0$ where $E(x, t)$ is defined by (15).

(3) $E(x, t)>0$ for $t>0$ is obvious by (15).

(4) We have

$$
E(x, t)=\frac{1}{(2 \pi)^{n}} \int_{R^{n}} \exp \left[-c^{2} t\left[\left(\sum_{i=1}^{p} \xi_{i}^{2}\right)^{3}+\left(\sum_{j=p+1}^{p+q} \xi_{j}^{2}\right)^{3}\right]^{k}+i(\xi, x)\right] d \xi
$$

put

$$
\begin{gathered}
\xi_{1}=y_{1}, \quad \xi_{2}=y_{2}, \ldots, \quad \xi_{p}=y_{p} \\
d \xi_{1}=d y_{1}, d \xi_{2}=d y_{2}, \ldots, d \xi_{p}=d y_{p}
\end{gathered}
$$

and

$$
\begin{gathered}
\xi_{p+1}=y_{p+1}, \quad \xi_{p+2}=y_{p+2}, \ldots, \quad \xi_{p+q}=y_{p+q} \\
d \xi_{p+1}=d y_{p+1}, \quad d \xi_{p+2}=d y_{p+2}, \ldots, d \xi_{p+q}=d y_{p+q}
\end{gathered}
$$

Thus ,we obtain

$$
\begin{gathered}
E(x, t)=\frac{1}{(2 \pi)^{n}} \int_{R^{n}} \exp \left[-c^{2} t\left[\left(\sum_{i=1}^{p} \xi_{i}^{2}\right)^{3}+\left(\sum_{i=1}^{p} \xi_{i}^{2}\right)^{3}\right]^{k}+i(\xi, x)\right] d y . \\
|E(x, t)| \leq \frac{1}{(2 \pi)^{n}} \int_{R^{n}} \exp \left[-c^{2} t\left(\left(\sum_{i=1}^{p} y_{i}^{2}\right)^{3}+\left(\sum_{j=p+1}^{p+q} y_{j}^{2}\right)^{3}\right)^{k}\right] d y .
\end{gathered}
$$

The same process as Lemma 2.1 then (21) becomes

$$
|E(x, t)| \leq \frac{1}{9.2^{n} \pi^{n / 2} k^{2}\left(c^{2} t\right)^{\frac{n}{6 k}}} \frac{\Gamma\left(\frac{p}{6 k}\right) \Gamma\left(\frac{q}{6 k}\right)}{\Gamma\left(\frac{p}{2}\right) \Gamma\left(\frac{q}{2}\right)}
$$


(5)We have

$$
E(x, t)=\frac{1}{(2 \pi)^{n}} \int_{R^{n}} \exp \left[-c^{2} t\left[\left(\sum_{i=1}^{p} \xi_{j}^{2}\right)^{3}+\left(\sum_{j=p+1}^{p+q} \xi_{j}^{2}\right)^{3}\right]^{k}+i(\xi, x)\right] d \xi
$$

Since $E(x, t)$ exists, then

$$
\begin{aligned}
\lim _{t \rightarrow 0} E(x, t) & =\frac{1}{(2 \pi)^{n}} \int_{\mathbb{R}^{n}} e^{i(\xi, x)} d \xi \\
& =\delta(x), \quad \text { for } \quad x \in \mathbb{R}^{n}
\end{aligned}
$$

See (R. Haberman,1983 , p396, Eq.(10.2.19b)). then

$$
\lim _{t \rightarrow 0} u(x, t)=u(x, 0)=\delta * f(x)=f(x)
$$

which satisfies (14)

\section{Acknowledgment}

The authors would like to thank The Thailand Research Fund and Graduate School, Chiang Mai University, Thailand for financial support.

\section{References}

A. H. Zemanian. (1965). Distribution Theory and Transform Analysis, McGraw-Hill, New York.

A. Kananthai. (1997). On the Solution of the n-Dimensional Diamond Operator. Applied Mathematics and Computational, 88:27-37.

A. Kananthai. (1999). On the Fourier Transform of the Diamond Kernel of Marcel Riesz. Applied Mathematics and Computation, 101:151-158.

F. John. (1982). Partial Differential Equations, $4^{\text {th }}$ Edition, Springer-Verlag, New York.

K. Nonlaopon, A. Kananthai. (2003). On the Ultra-hyperbolic heat kernel. Applied Mathematics, Vol. 13, No. 2, $215-225$.

R. Haberman. (1983). Elementary Applied Partial Differential Equations, $2^{\text {nd }}$ Edition, Prentice-Hall International, Inc. 